\title{
CONTACTS DE LANGUES ET MULTIMODALITE CHEZ DES LOCUTEURS SOURDS : CONCEPTS ET OUTILS METHODOLOGIQUES POUR L'ANALYSE
}

\author{
Agnès Millet \& Isabelle Estève ${ }^{1}$ \\ Laboratoire Lidilem, Université Stendhal-Grenoble3
}

\begin{abstract}
Résumé
Cette contribution propose des outils de transcription et d'analyse des pratiques langagières de locuteurs sourds, dont les discours sont inscrits le plus souvent dans le bilinguisme, le contact de langues et la bimodalité. Réalisée sous le logiciel ELAN®, la grille hiérarchique que nous proposons et dont nous détaillons tous les niveaux d'analyse autorise des approches qualitatives et quantifiées des observables. Elle permet de rendre compte de la diversité des moyens utilisés (verbal/non verbal ; vocal/gestuel), de leur combinatoire et de leurs interactions. Elle permet également d'envisager des indices de complexité syntaxique et discursive, qui se construisent dans cette bilingualité particulière ancrée d'entrée de jeu dans la bimodalité. Les visées sont théoriques et descriptives et doivent trouver des prolongements psycholinguistiques et didactiques.
\end{abstract}

La présente contribution s'inscrit dans un projet ${ }^{2}$ plus large qui vise à rendre compte des conduites narratives des enfants, dans une optique développementale, en interrogeant la part de la multimodalité dans les processus acquisitionnels du langage (Colletta, 2004). Les divers corpus recueillis visent à tester des variables liées à la culture d'origine des enfants (des équipes italiennes et états-uniennes sont intégrées au projet) ainsi que des variables liées à la typicité du développement langagier (des enfants ordinaires, des dysphasiques et des sourds ont été sollicités). Les outils d'analyse proposés ici se sont forgés à partir d'une réflexion préalable menée par les différentes équipes associées au projet concernant les enfants normo-entendants (Colletta et al., 2008), et si, pour des raisons de possibilité de comparaisons, certains éléments - spécialement pour ce qui concerne la description de l'utilisation de la langue française - ont été conservés à l'identique, la plupart des concepts proposés pour la description ont dû être revus, réaménagés, augmentés pour répondre à la spécificité des conduites langagières des enfants sourds, qui s'ils sont tout comme les enfants entendants ancrés dans la bimodalité, ont une utilisation particulière de la modalité gestuelle, qui peut, pour certains, revêtir des dimensions linguistiques.

\footnotetext{
1 agnes.millet@u-grenoble3.fr ; isabelle.esteve@u-grenoble3.fr

2 Projet, dirigé par Jean-Marc Colletta, financé par l'ANR (Agence Nationale de la Recherche, organisme français de financement public sur projet de recherche), intitulé « L'acquisition et les troubles du langage au regard de la multimodalité de la communication parlée » et courant sur les années 2005-2009.
} 


\section{Introduction}

\subsection{Objectifs}

Ainsi, la grille d'analyse que nous proposons est le fruit d'une réflexion qui vise à décrire et à comprendre les pratiques communicatives des locuteurs sourds dans leurs aspects bilingues et multimodaux. D'une manière générale, le bilinguisme sourd est dit «bimodal » parce qu'il allie une langue vocale (ici, le français) et une langue gestuelle (ici, la Langue des Signes Française - LSF). Ces deux langues recourent à deux canaux de production différents, une configuration articulatoire qui offre au locuteur sourd bilingue la possibilité de produire des énoncés dans les deux langues simultanément.

Mais les langues ne sont pas seules en cause puisque verbal et non-verbal s'étayent dans l'expression des sujets parlants. Ainsi, pour décrire la bimodalité dans toutes ses dimensions, nous nous placerons à l'échelle du discours, considérant l'expression du locuteur sourd comme un tout, où interviennent deux modalités : les modalités vocale et gestuelle. Nous proposons ainsi d'analyser les ressources communicatives du locuteur sourd à l'échelle du langage dans son acception large telle que proposée par Mc Neill ${ }^{3}$. Les visées de la présente étude sont essentiellement théoriques et descriptives, mais des prolongements psycholinguistiques - en terme de compréhension du développement du langage chez l'enfant sourd - ainsi que des prolongements didactiques - en terme de prise en compte de la multimodalité et du plurilinguisme tel que défini par le Conseil de l'Europe ${ }^{4}$ dans l'enseignement aux enfants sourd - sont d'ores et déjà à l'étude.

Face à la complexité des phénomènes observés, on a posé que les énoncés auxquels nous avons été confrontées sont composés de deux messages : un message vocal et un message gestuel, chacune des modalités pouvant être utilisées dans une dimension verbale ou non-verbale - à savoir français et onomatopées pour la modalité vocale, LSF et gestes non-verbaux pour la modalité gestuelle.

\subsection{Contexte et genèse}

Les études sur l'utilisation de la multimodalité chez les sourds sont extrêmement rares. On notera quelques études spécifiques (Loncke, Quertinmont, Martens \& Dussart, 1996; Lucas, 2001), une étude menée sur les adultes entendants nés de parents sourds ${ }^{5}$ (Emmorey, Borinstein \& Thompson, 2005) et quelques études sociolinguistiques générales qui y font allusion (Lucas, 1996 ; Lucas, Bayley \& Valli, 2001 ; Vonen, Arnesen, Enerstvedt \& Nafstad, 1996). Toutes ces études cependant ne s'en tiennent le plus souvent qu'à la bimodalité linguistique (langue vocale, langue gestuelle), une restriction que la fréquentation récente de corpus enfantins nous a amenées à dépasser.

En effet, deux corpus sont à l'origine des développements théoriques et méthodologiques présentés ici. Le premier, recueilli en 2006, auprès d'un groupe de pairs composé de huit jeunes adultes sourds ${ }^{6}$, dont les analyses (Estève, 2006, 2007 ; Guigas, 2006 ; Millet, 2007 ; Millet \& Estève, 2008 ; Millet, Estève \&

${ }^{3}$ «[...] gestures are an integral part of language as much as are words, phrases and sentences - gestures and language are one system » (Mc Neill, $1992: 2$ ).

4 «[...] l'approche plurilingue met l'accent sur le fait que, au fur et à mesure que l'expérience langagière d'un individu dans son contexte culturel s'étend de la langue familiale à celle du groupe social puis à celle d'autres groupes [...], il/elle ne classe pas ces langues et ces cultures dans des compartiments séparés mais construit plutôt une compétence communicative à laquelle contribue toute connaissance et toute expérience des langues et dans laquelle les langues sont en corrélation et interagissent » (Conseil de l'Europe, 2005 : 11).

5 dits « CODAs » pour Children of Deaf Adults.

${ }^{6}$ Les recueils on été effectués auprès d'un groupe de pairs composé de 5 filles (Zoé, Léa, Nina, Alix et Eva) et de 3 garçons (Gil, Tim et Pat). Deux ont des parents sourds (Nina et Gil). Les parcours scolaires sont très divers. Les données vidéo obtenues sont les suivantes : $2 \mathrm{~h} 40 \mathrm{mn}$ d'entretiens effectuées auprès de chacun des participants, à des fins d'étude des représentations, à quoi s'ajoutent $4 \mathrm{mn}$ pour Tim dans 2 autres situations; $15 \mathrm{mn}$ pour Léa dans 4 autres situations ; $10 \mathrm{mn}$ pour Eva dans 2 autres situations. La disparité des corpus est liée au fait que nous avons préféré, pour les situations autre que celle de l'entretien, laisser les personnes se filmer elles-mêmes pour obtenir des données écologiques. 
Guigas, 2008) ont permis une première série d'élaborations théoriques que nous exposerons dans le premier paragraphe. Le second, en cours de recueil et d'analyse, constitué auprès d'enfants sourds ${ }^{7}$, a donné lieu à des analyses partielles (Batista, 2008 ; Estève, 2008) et nous a permis de mettre au point une grille de transcription - et donc d'analyse - sous le logiciel ELAN $\AA^{8}$ qui permette à la fois de produire des résultats quantifiés (proportion des langues et des modalités par exemple) et des extractions de corpus visant des analyses plus qualitatives (interprétation des changements de langues et de modalités par exemple).

La confrontation des deux corpus et des diverses analyses, nous amène aujourd'hui à proposer un outil de description des pratiques bilingues et bimodales des locuteurs sourds qui puisse rendre compte d'un ensemble de phénomènes discursifs spécifiques. Cet outil se présente sous la forme d'une grille hiérarchisée, comme le permet le logiciel utilisé. Les références aux lignes de notre grille - appelées « acteurs », dans la terminologie du logiciel - seront notées en italique dans la suite du texte. On trouvera l'intégralité de la grille en annexe, l'ensemble de cet article visant à expliciter et argumenter nos choix d'analyse.

\subsection{Quelle unité de description?}

Sur la première ligne des annotations appelée enfant nous repérons temporellement l'unité fondamentale de description. Pour les entendants, dans notre recherche, ces repérages de séquences passent par une identification du groupe de souffle et/ou des propositions. Concernant les personnes sourdes, les repérages de groupes de souffle nécessiteraient des technologies (capteurs, etc.) que nous n'avons pu mettre en œuvre. Nous nous en tiendrons donc à un découpage de type propositionnel - i.e. autour d'un verbe. Cependant, s'agissant des productions des enfants, il est vite apparu que la notion de proposition n'était pas toujours opératoire, puisque bien des enfants ne s'expriment pas dans des dimensions strictement linguistiques. En effet, certains enfants allient le linguistique et le non linguistique pour parvenir à restituer l'histoire qu'ils veulent raconter. La description doit alors prendre en compte le «vouloir dire» (Lüdi \& Py, 1986) du locuteur, et ce, que l'expression passe par des dimensions linguistiques ou non linguistiques. En effet, sans être engagé dans une structuration strictement syntaxique - qui appartient aux systèmes linguistiques - le locuteur est néanmoins engagé dans une structuration sémantique qui s'appuie sur le non-verbal qu'il convient de prendre en compte.

Dans l'exemple suivant, il nous apparaît que Abdel $^{9}$, âgé de 6 ans, par la conjugaison bimodale des ressources non-verbales (onomatopées et gestes) reliées à une forme de thématisation exprimée en français (phénomène sur lequel nous reviendrons plus en détail plus loin), exprime une unité sémantique, référant à un segment d'épisode de l'histoire proposée à la narration.

Français : et le bébé oiseau et...et dedans le bébé

Gestes: MG (index tendu) mvt latéral de G>D MG (index tendu) MD (conf.coquille) mvt de recouvrement

Fançais : oiseau

Gestes : $\quad$ MG (index tendu) poursuit trajet latéral et revient en position initiale $\mathrm{G}$

$\mathrm{MG}=$ main gauche, $\mathrm{G}>\mathrm{D}=$ de la gauche vers la droite, $\mathrm{mvt}=$ mouvement, conf $=$ configuration manuelle

7 Conformément à la méthodologie générale de la recherche, des enfants sourds scolarisés dans les agglomérations lyonnaise et grenobloise ont visionné 2 minutes 46 d'un petit dessin animé de Tom et Jerry et nous l'on raconté ensuite. Nous possédons actuellement les narrations de 15 enfants scolarisés dans des structures " oralistes », où néanmoins la LSF est présente dans l'environnement de l'enfant, et 9 enfants scolarisé dans des structures « bilingues ». Les enfants sont âgés de 5 à 11 ans. Tous les exemples donnés ici proviennent de la restitution narrative du petit dessin animé.

${ }^{8}$ Elan est un logiciel libre qui permet d'aligner les corpus video-audio en indexant temporellement des lignes ad hoc que l'on relie au déroulement de l'image et du son (http://www.mpi.nl/tools/elan.html ).

9 Tous les prénoms de tous les corpus ont été changés.

Journal of Language Contact - VARIA 2 (2009)

www. jlc-journal.org 

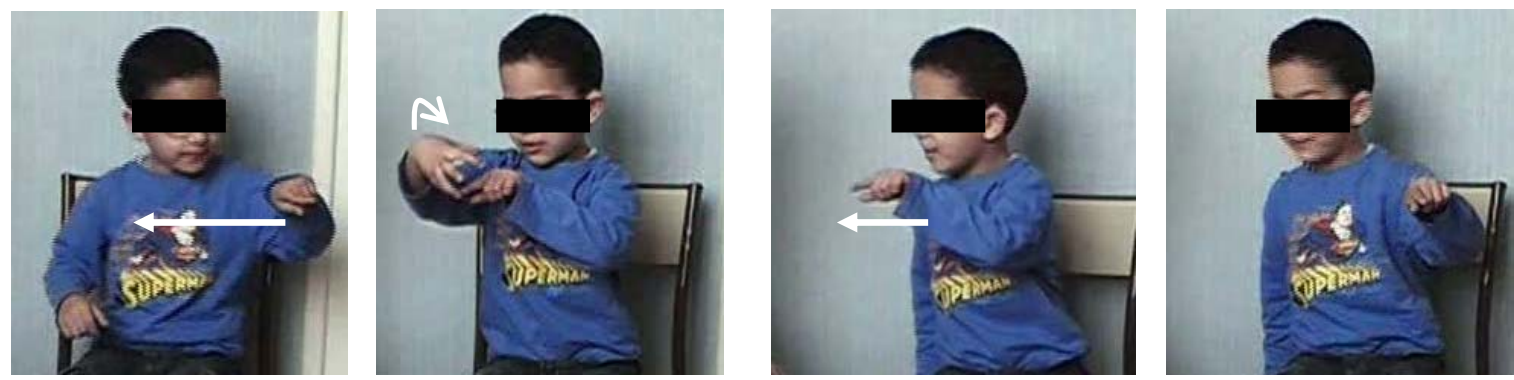

Ainsi, lorsque l'expression n'est pas strictement linguistique et que le découpage en proposition est impossible, et malgré les difficultés liées à une indéniable part interprétative, l'unité retenue sera une unité sémantico-syntaxique, ordonnée autour de l'idée de la restitution d'un procès quel que soit le moyen utilisé.

Comme on le voit dans notre exemple, la description des conduites langagières a nécessité la prise en compte de quatre dimensions qui constituent les outils fondamentaux de la description, que nous avions déjà utilisés dans notre étude sur les adultes.

\section{Outils fondamentaux}

En effet, tant dans les corpus concernant les adultes que dans ceux concernant les enfants, on observe une utilisation, dans des proportions variées selon les individus, de toutes les ressources langagières à disposition des locuteurs en terme de langue (langue française, LSF) et en terme de modalité (vocale ou gestuelle) qui nécessite d'être transcrites comme données de premier ordre.

\subsection{Quatre lignes majeures}

Ainsi, notre grille d'annotation s'organise au premier chef à partir de quatre lignes majeures correspondant aux deux langues et aux deux modalités en présence, inscrites sous la ligne enfant. De façon évidente, elles s'intitulent français, onomatopées, $L S F$, gestes. Elles constituent le premier niveau hiérarchique de la description. Des conventions de transcription que nous ne pouvons développer dans le détail ici on été établies pour les remplir. Aussi nous contenterons-nous de donner un exemple de transcription pour chacune d'entre elles avec quelques commentaires :

- français : l'intégralité des propos du locuteur est annotée en respectant la prononciation, les pauses, les hésitations, les ruptures de syntagmes, etc , comme par exemple « la... la... la maman de *zoiseau / i(l) i(l) travaille »;

- onomatopées : il s'agit de transcrire au mieux les sons et les bruitages vocaux produits : « chuuuo ::: ahahaa :::: , pchchchcttt »;

- $L S F$ : les conventions de transcriptions, liées à l'analyse linguistique que l'on peut faire de cette langue sont développées au point 5.2, notons d'ores et déjà que, comme c'est l'usage, les signes de la LSF sont notés en majuscule par la traduction centrale que l'on peut en faire en français : « OISEAU ๕UF pr-œuf ${ }^{10}$, FAIREUN-BOND 》;

- gestes : il s'agit de décrire, dans une glose la plus exhaustive possible, les gestes effectués : «Deux mains (conf.pince) mvt de préhension $\mathrm{G}>\mathrm{D}$ (mime couvrir) ».

Comme nous le verrons plus loin, les réflexions menées sur les corpus enfantins nous ont amenées, dans la perspective de pouvoir répondre à la question du développement langagier, à les enrichir de manière à pouvoir rendre compte des outils syntaxiques et discursifs mis en œuvre par les locuteurs. Auparavant, il convient de discuter le statut d'une cinquième ligne qui fait débat.

${ }^{10}$ « pr-œuf » renvoie à « proforme manuelle » référant à l'œuf. Pour la définition des proformes voir note 14. 


\subsection{Une cinquième ligne délicate}

Une ligne supplémentaire nous est apparue en effet nécessaire : celle appelée labialisation, qui pose de redoutables problèmes théoriques. En effet, les labialisations sont, lors d'un discours en LSF, une forme d'articulation, sans émission sonore, du mot français correspondant au signe produit. Certains chercheurs (Séro-Guillaume, 1994) estiment que ces labialisations font partie intégrante de la LSF, et spécialement lorsqu'elles permettent de discriminer sémantiquement des signifiants identiques en LSF, mais différenciés en français, s'apparentant en cela aux initialisations ${ }^{11}$. Les deux phénomènes sont en effet dus aux contacts de langues, mais si les initialisations sont totalement intégrées dans la formation même du signe de la LSF, puisqu'elles en conditionnent la forme de main, les labialisations nous semblent plutôt s'inscrire dans une pratique bilingue français/LSF et monomodale, puisque, aucun son n'étant émis, les labialisations nous paraissent relever de la modalité gestuelle : elles s'opposent en cela aux vocalisations (prononciation effective des mots français) qui introduisent le sujet parlant dans la modalité vocale ${ }^{12}$.

\section{Les types de pratiques}

L'étude de la combinatoire de ces premières dimensions permet de caractériser les types de pratiques. Les questions qui se posent sont alors diverses et concernent les critères permettant de caractériser l'utilisation des langues et des modalités - spécialement les critères permettant de catégoriser les propositions présentes dans les discours au-delà d'un simple croisement modalité/langue (par exemple monolingue monomodal / bilingue bimodal / monolingue bimodal). En effet, d'une part, même si certains discours d'adultes peuvent être considérés comme bilingues et donc par définition comme a priori bimodaux, la réalité linguistique fait que très souvent une des deux langues prédomine. A cet effet, nous avons pris le parti de considérer, à l'échelle de notre unité de description, que lorsqu'il y a utilisation de la voix pour une expression en français, de manière continue sur une unité, l'énoncé était à « base français ». Les pratiques à « base français » constituent ainsi le premier pôle d'un continuum d'expression langagière qui se définit et se décline de la façon suivante.

\section{1. les pratiques à « base français »}

Les pratiques à « base français » regroupent donc les propositions structurées par la langue française avec émission sonore dans lesquelles on va trouver des utilisations variables de la LSF et/ou des gestes, que nous avons été amenées à catégoriser. Les distinctions opérées constituent autant de catégories venant enrichir la ligne base français comme suit :

- français monolingue monomodal : uniquement en français vocal, sans gestes ;

- français monolingue bimodal : utilisation de la langue française et d'une gestualité non linguistique ;

- français bilingue avec insertions ponctuelles de LSF : le français est utilisé de manière continue avec quelques signes de la LSF ;

\footnotetext{
${ }^{11}$ L'initialisation consiste à choisir pour un signe de la LSF une forme de main qui représente la première lettre du mot français correspondant. Ces formes de lettres sont disponibles dans ce que l'on nomme «alphabet dactylologique ». Ainsi les signes [VACANCE] et [REPOS] ne sont distincts que par leur forme de mains renvoyant à la lettre « V » pour le premier et à la lettre « $\mathrm{R} »$ pour le second.

${ }^{12} \mathrm{Il}$ existe cependant des labialisations qu'on appellera figées, car elles sont régulièrement associées à des signes. On peut peut-être alors considérer qu'elles intègrent la LSF, dans la mesure où elles sont d'une utilisation très systématisée, cependant ces labialisations parce qu'elles sont justement figées ne correspondent pas nécessairement à la traduction qui serait faite en contexte ; c'est par exemple le cas de [FINI] qui est très régulièrement associé à (fini) mais qui ne se traduit que très rarement par «fini » en français. Ces labialisations figées se rapprochent alors des sons émis de manière récurrente avec certains signes, comme par exemple [SPECIFIQUE] - (pi). On peut alors discuter leur statut linguistique. Néanmoins on distinguera entre labialisations figées - avec un statut semi-linguistique - et onomatopées - qui introduisent dans la bimodalité et n'ont aucun statut linguistique, mais attestent que pour les sourds, la voix peut investir un statut non linguistique, associé à un signe gestuel linguistique, sans qu'il s'agisse de l'expression d'une émotion particulière (Millet, 2007).
} 
- français bilingue bimodal : français et LSF sont utilisés en continu, mais les signes de la LSF suivent la structure de la langue française - ce que l'on nomme souvent « français signé ».

\subsection{Les pratiques à « base LSF »}

A l'autre pôle du continuum se trouvent les pratiques à « base LSF », c'est-à-dire les propositions dans lesquelles la langue qui structure l'échange est la LSF. Tout comme les pratiques à « base français », elles présentent plusieurs variétés que nous avons glosées comme suit :

- LSF monolingue monomodal : utilisation de la seule LSF ;

- LSF monolingue bimodal : utilisation simultanée de la LSF et de sons (onomatopées, bruitages) ;

- LSF bilingue monomodal : utilisation de la LSF et de labialisations ;

- LSF bilingue avec insertions ponctuelles de frs : utilisation de la LSF avec quelques mots de français oralisés ;

- LSF bilingue bimodal : utilisation de la LSF avec alternance vocalisations/labialisations : le français est utilisé en continu mais dans deux modalités.

\subsection{Les pratiques à « base bilingue»}

$\mathrm{Au}$ centre du continuum se situent les pratiques à "base bilingue ${ }^{13}$ qui correspondent à des unités sémantico-syntaxiques dont la base organisatrice n'est pas attribuable à une langue en particulier, mais à l'interaction des deux langues. Autrement dit, dans ce cas, la structure de l'énoncé relève pour partie des structures de la langue française et pour partie des structures de la LSF. Ces formes de code mixing peuvent se construire dans la simultanéité comme dans l'exemple suivant tiré du corpus recueilli auprès des adultes, chez lesquels ce type de pratiques est fréquent :

Alix

Français : moi je fais beaucoup différentes écoles

LSF : [PTE-1] [FAIRE][BEAUCOUP][DIFFÉRENTES][ECOLES]

ou dans la séquentialité comme le montre cet autre exemple, tiré du corpus recueilli auprès des enfants, ces pratiques n'étant pas attestées dans notre corpus d'adultes.

\section{Abdel}

Français : et après la souris... la souris

$L S F$ :APRES pr-corp-souris, DORMIR

\subsection{Les pratiques à « base non-verbale»}

En marge de ce continuum, l'étude des narrations enfantines nous a amenées à envisager, pour les segments qui sont structurés par des éléments non linguistiques, une base non-verbale qui se décline de la façon suivante :

- gestuel: référant aux pratiques composées uniquement de gestes non-verbaux ;

- vocal : référant aux pratiques composées uniquement d'onomatopées ;

- bimodal : référant aux pratiques composées de gestes et d'onomatopées.

On soulignera que nous avons choisi de catégoriser comme non-verbales des pratiques langagières pour lesquelles l'amorce du groupe sémantico-syntaxique, via un connecteur ou une précision

${ }^{13}$ Dans notre première étude (A Millet \& Estève, 2008) nous parlions de « code-blends intégraux ». Le terme « codeblend» est emprunté à K. Emmorey, qui les définit comme des productions simultanées en American Sign Language et en anglais (Emmorey et al., 2005). Ce terme de « code-blend» impliquant la simultanéité, le terme de « base bilingue » nous a paru préférable car moins restrictif. 
linguistique, de type « après » ou « comme ça » par exemple, était réalisée en français ou en LSF - ces éléments linguistiques nous ayant paru tenir un simple rôle d'« impulseur propositionnel ».

\subsection{Applications : établir des profils et rendre compte des dynamiques du continuum}

Dans notre étude sur les adultes (Millet \& Estève, 2008), il nous a été ainsi possible de caractériser différents profils, dont nous reprenons ici deux exemples contrastés qui nous permettront ensuite de montrer la dynamique des déplacements langagiers sur le continuum.

\subsubsection{Variation inter-individuelles en situation d'entretien}

En situation d'entretien avec un interlocuteur entendant maîtrisant la LSF, par exemple, on a pu observer que les types de pratiques adoptées par Tim et Eva dénotent une exploitation différente des langues et des modalités.

Dans ce cas, les variations inter-individuelles témoignent, de manière caractéristique, d'une orientation linguistiquement différenciée de ces deux locuteurs comme le montre la figure 1 ci-après. Face à un interlocuteur entendant bilingue, les stratégies d'adaptations communicatives de Tim et Eva sont, en effet, strictement opposées. Tim choisit d'interagir en ne sollicitant que la modalité gestuelle : il utilise en continu la LSF et les labialisations, adoptant donc majoritairement des pratiques à base LSF bilingues monomodales (près de 90\%). Eva, quant à elle, adopte principalement des pratiques structurées par le français, où les deux langues sont utilisées en continu - i.e. français bilingue bimodal (plus de $70 \%$ ).

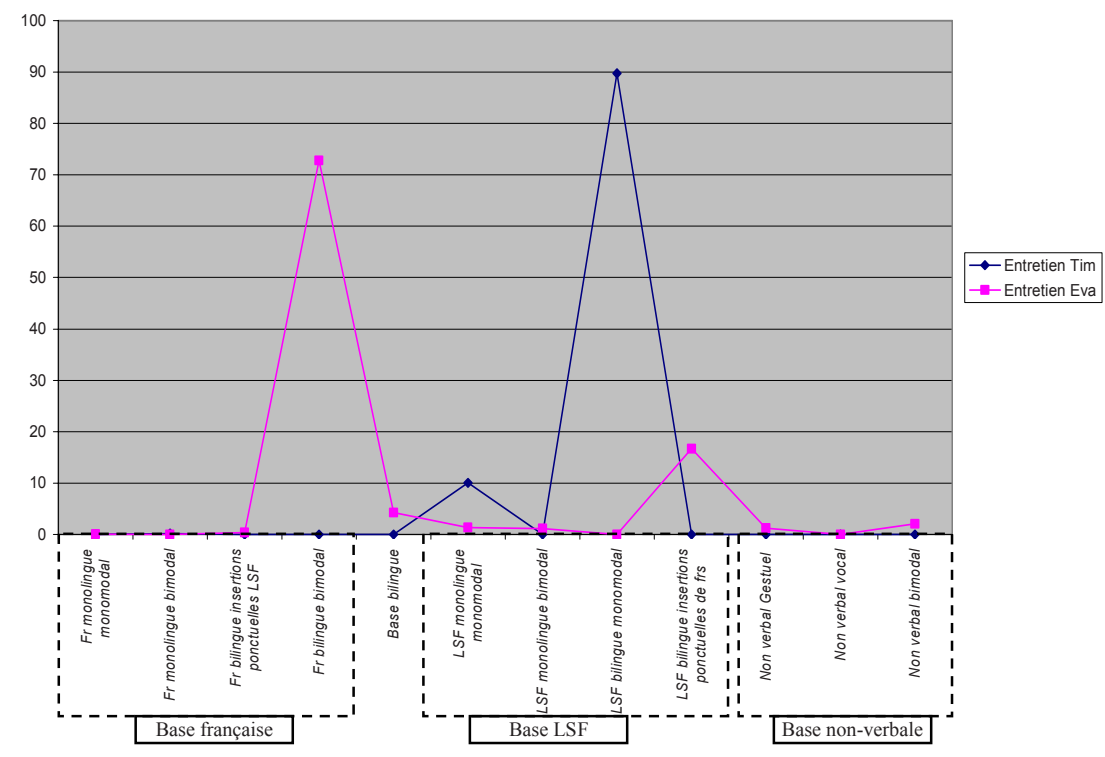

Figure 1. Pratiques langagières de Tim et Eva en situation d'entretien

Dans cette situation de communication se dessinent ainsi deux profils différents de locuteurs sourds, profils qui se précisent dans l'adaptation communicative observée dans les autres situations de communications informelles recueilles : à savoir en famille, et entre amis. 


\subsubsection{Profil à base gestuelle vs profil bilingue}

Comme le montre la figure 2, Tim présente un profil à base gestuelle. En effet, ses pratiques, quelle que soit la situation, sont marquées par une sollicitation constante de la modalité gestuelle : sous forme de labialisations dans son parler à base LSF - comme nous venons de le voir -, sous forme de gestualité nonverbale dans une adaptation communicative à un locuteur entendant monolingue et enfin sous forme d'une utilisation alternée de pratiques à base bilingue et de discours à base LSF avec sa famille entendante.

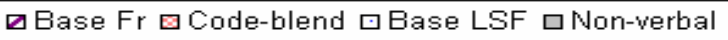

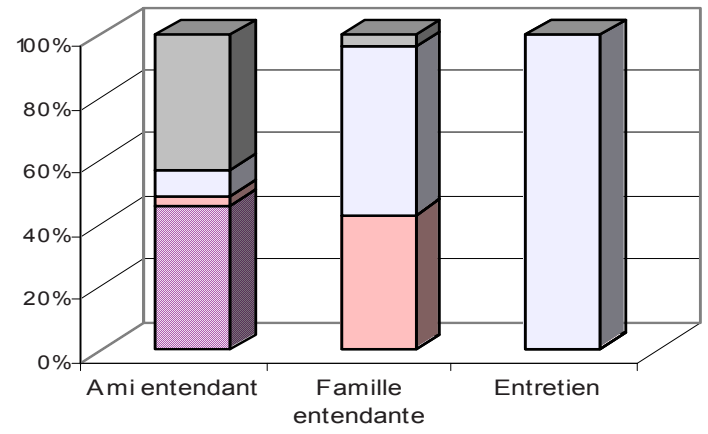

Figure 2. Adaptations communicatives de Tim

Eva présente, quant à elle, un profil, qui peut être caractérisé comme étant bilingue, ainsi que le montre la figure 3. En effet, chez cette locutrice, quelles que soient les compétences en LSF de ses interlocuteurs entendants, les tours de parole sont dominés par l'utilisation d'un parler bilingue à base français. Avec son amie sourde, on note que la LSF est adoptée comme base de ses discours, mais ses pratiques restent fondamentalement bilingues, puisque seules $10 \%$ des propositions sont des pratiques LSF monolingues.

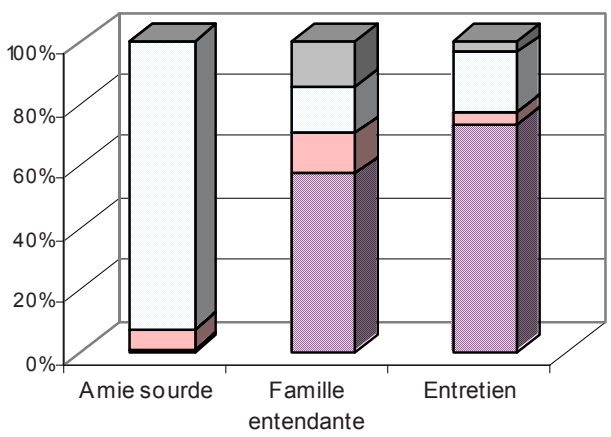

Figure 3. Adaptations communicatives de Eva 


\subsubsection{Situer les locuteurs sur le continuum bilingue}

L'adaptation communicative que mettent en œuvre Tim et Eva relève ainsi de dynamiques langagières différentes et les stratégies communicatives différenciées des deux locuteurs permettent dès lors de les situer en terme de chemin/distance parcouru(e) le long d'un continuum langagier aux extrémités duquel se trouvent des pratiques monolingues en français et monolingues en LSF. Entre ces pôles monolingues, s'inscrivent toutes les pratiques intermédiaires composant le " pôle bilingue ».

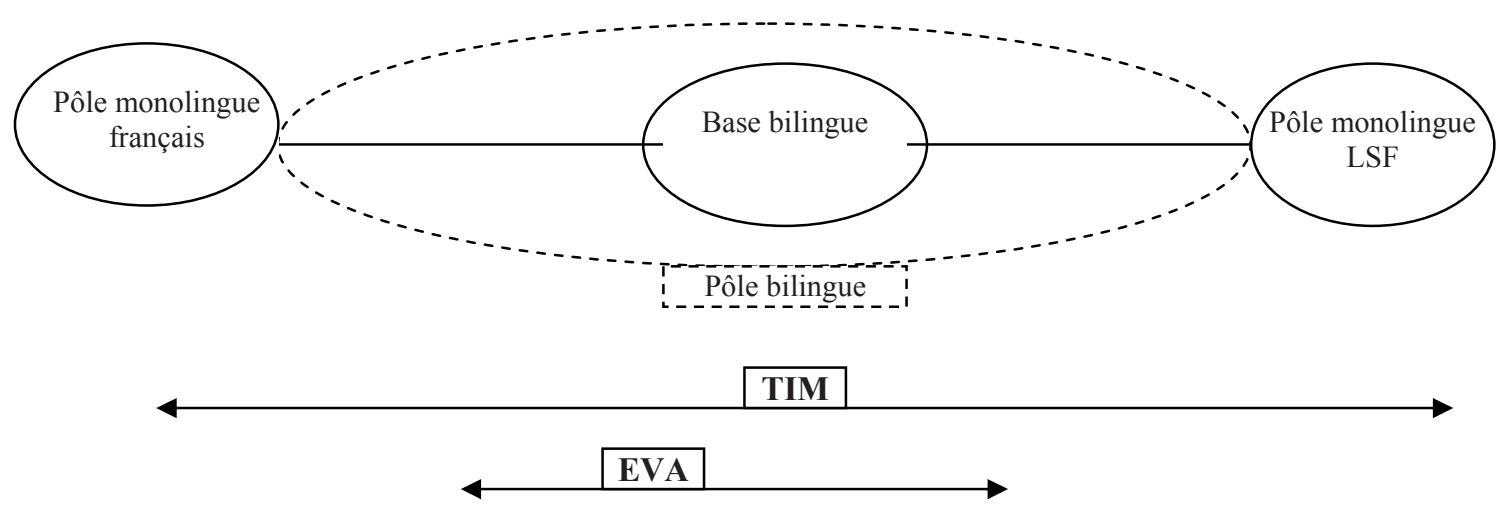

Figure 4. Répertoires communicatifs de Tim et Eva

L'adaptation communicative mise en œuvre par Tim témoigne d'un recours à la quasi-totalité des ressources communicatives rendues possibles par la bimodalité, tandis que Eva semble avoir une difficulté à se placer exclusivement dans l'une ou l'autre langue. L'adaptation communicative dont fait preuve cette locutrice est circonscrite aux pratiques situées au cœur du "pôle bilingue », dans une utilisation variable de la dimension verbale de chaque modalité. Cette locutrice caractérise fortement la particularité du bilinguisme sourd par le recours constant à la bimodalité à travers toutes les situations de communications.

Par ces deux exemples, nous avons voulu donner un aperçu de la façon dont les annotations portées sous ELAN ${ }^{\circledR}$ peuvent être exploitées à ce premier niveau général des pratiques langagières.

Cependant, la description et l'analyse des corpus recueillis auprès des enfants, nous ont amenées à envisager d'autres annotations à même de rendre compte de la structuration souvent plus complexe de leurs discours - complexité que nous supposons liée au fait que leurs ressources langagières sont en construction. Nous avons en outre souhaité que ces annotations soient susceptibles, à terme, de nous fournir des données exploitables, pour décrire, dans des études longitudinales ou transversales, le développement langagier de ces enfants, pour la mesure duquel on ne dispose actuellement que de très peu d'instruments.

\section{Un outil d'analyse rendant compte de la complexité}

La construction langagière des enfants semble en effet, à des degrés divers, s'appuyer sur les deux modalités et les deux langues. Si certains enfants paraissent s'ancrer dans un pôle monolingue du continuum (français oral ou LSF), bon nombre d'entre eux jouent sur les langues et les modalités pour construire leurs discours - leur répertoire communicatif étant alors mobilisé dans toutes ses ressources pour les besoins de l'expression. La question se pose donc de rendre compte, à l'échelle de l'unité choisie, de l'organisation globale de la production en envisageant, en terme de rapport intersémiotique, les relations entre les ressources mobilisées. 


\title{
4.1. Restituer la complexité
}

En effet, la spécificité des stratégies narratives des enfants sourds se caractérise par la multiplicité des ressources combinées au cours d'une même unité sémantico-syntaxique. Ainsi, la complexité des discours des enfants sourds ne peut s'évaluer uniquement à l'échelle linguistique, mais dans l'appréhension langagière de l'organisation de chaque unité discursive. Nous l'illustrerons en examinant la façon dont les enfants les plus jeunes - de 5 à 7 ans - articulent le thème et le rhème dans leurs énoncés, en nous centrant sur deux exemples.

\subsubsection{L'élaboration de la relation thème/rhème}

Dans le premier exemple, où l'expression s'inscrit dans des pratiques à base français, le thème est énoncé en français, tandis que le rhème est assuré dans une combinaison bimodale onomatopées/gestes.

\author{
Noah \\ Français : $\quad$ le petit oiseau et là \\ Onomatopées: $\quad$ poum poum poum poum poum poum \\ Gestes: deux mains jointes (conf.boule) rebondissent en différents points de l'espace
}

Noah, âgé de 7 ans, s'attache ici à décrire l'épisode où l'oiseau fait des bonds dans le nid. L'interaction des ressources verbales et non-verbales confère à cette unité un sens complexe se composant d'éléments qui pris isolément n'auraient que peu de sens. Chacune des ressources mobilisées endosse un rôle tout à la fois spécifique et complémentaire dans l'élaboration de la relation thème/rhème que nous pourrions traduire ainsi : «y a un petit oiseau, et soudain il fait plein de bonds partout».

Dans le deuxième exemple, les deux langues, dans une pratique bilingue donc, sont utilisées pour exprimer simultanément le thème et le rhème puis les onomatopées viennent spécifier le rhème.

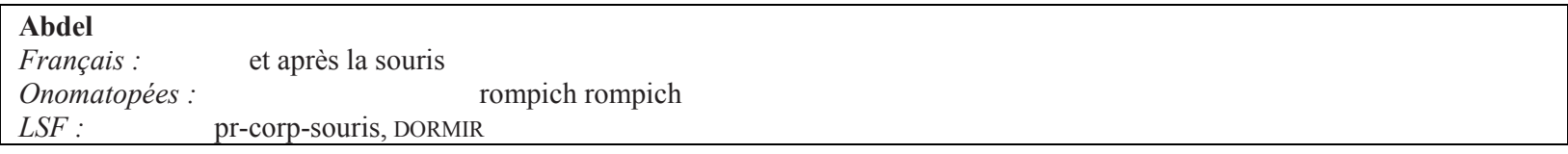

Dans cette construction particulière, chaque ressource est utilisée pour un rôle particulier : le français pour identifier l'actant du procès, la LSF pour représenter le procès, tandis que les onomatopées endossent un rôle que l'on pourrait caractériser comme adverbial ou participial : la souris dort en ronflant. Si dans cet exemple, ces différentes productions sont signifiantes en elles-mêmes, aucune n'est porteuse à elle seule du sens global qu'acquiert l'unité par l'interaction sémantique et structurelle de ces différentes ressources dans l'élaboration de la relation thème/rhème.

Les discours des enfants sourds semblent ainsi manifester une tension des pratiques vers la dimension non-verbale que l'on n'observe que très rarement chez les adultes, et qui se manifeste bien au-delà de la seule relation thème/rhème, que nous venons d'évoquer. En effet, les unités sémantico-syntaxiques s'organisent en une série de segments, présentant une imbrication, dans l'utilisation et la conjugaison des langues et des modalités, dont il faut bien rendre compte.

\subsubsection{Composition des unités sémantico-syntaxiques}

Les unités sémantico-syntaxiques reposant sur la mobilisation des ressources mixtes - i.e verbales et non-verbales - sont ainsi assez fréquentes chez les enfants sourds, et adoptent des configurations combinatoires et sémantiques diverses. Nous nous attarderons ici sur les extraits d'une seule narration, celle d'Abdel, pour illustrer la variété des stratégies rencontrées dans les discours d'un même locuteur. 
Dans une structure bimodale à base LSF, la modalité vocale peut être utilisée successivement dans ses dimensions non-verbales et verbales.

\begin{tabular}{lc}
\hline Français : & fleur \\
Onomatopées : & pfuuuu ::: \\
LSF : & stf-œuf, TOMBER, PLUS-BAS
\end{tabular}

Dans cet exemple, Abdel décrit la chute de l'oiseau. Le recours à la modalité vocale vient compléter le schéma spatial élaboré en LSF, les onomatopées sont utilisées pour spécifier l'aspect lent et continu de la chute, et le recours ponctuel au français présente l'aboutissement de la chute de l'œuf : dans la fleur.

Dans une structure bimodale à base français, le recours à une symbolisation bimodale non-verbale peut s'inscrire dans une stratégie compensatrice d'un manque lexical en français, comme l'illustre l'exemple suivant.

\begin{tabular}{|lc|}
\hline$F:$ et puis... i(l)...i(l) & des grosses \\
$O::$ & avec \\
$G:$ Deux mains écartées autour de la tête MD mut en dôme autour de la tête MD (index tendu) tracé en pics devant le \\
front MD (index tendu) fini le tracé en arc tour de la tête
\end{tabular}
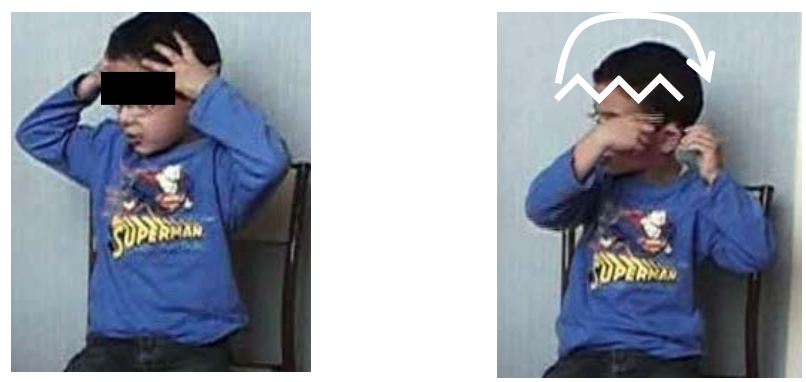

Ici, Abdel, confronté au manque de vocabulaire pour le mot « coquille », procède à la description de la coquille cassée autour de sa tête, par le recours aux gestes et aux onomatopées en complément du français. Cette stratégie illustre ses capacités expressives et ses compétences langagières de symbolisation, qui vont bien au-delà de ses compétences strictement linguistiques.

Les constructions où la modalité gestuelle est mobilisée successivement dans ses dimensions verbales et non-verbales sont également nombreuses, du fait que le système linguistique de la LSF est en cours d'acquisition. Ainsi, la complexité de la structure linguistique que l'enfant cherche à construire peut amener l'apparition de gestes non-verbaux.

Français: et l'oiseau... et l'oiseau ... euh bébé oiseau.... oh puis..

LSF : pr-corp-souris, DORMIR -

Gestes: $\quad$ MD (index tendu) mvt sineux dans le dos

Dans cette structure, qui démarre sur un mode bilingue, Abdel tente de décrire l'action de l'œuf, et de la souris simultanément, l'œuf roulant jusque sous la souris. La complexité de la structure linguistique correspondante en LSF amène l'apparition d'un geste non-verbal pour symboliser le trajet de l'oeuf parallèlement à la prise de rôle en LSF qu'adopte Abdel - en continu, ce que marquent les pointillés dans notre exemple - pour représenter la souris en train de dormir. Cet exemple, qu'on pourrait traduire par «l'œuf roule jusque sous la souris qui est en train de dormir», semble relever d'avantage d'une 
symbolisation linguistique en cours de construction, que d'une stratégie consciente de recours aux ressources non-verbales.

Ainsi, au regard de la multiplicité des structures possibles, dont nous venons de donner un aperçu, il semble nécessaire de concevoir des outils de description qui puissent rendre compte à la fois de la nature des ressources combinées et des rapports intersémiotiques qui régissent leurs interactions. En maintenant dans la description une perspective à la fois combinatoire et sémantique, nous serons en effet en mesure de saisir le rôle qu'endosse chaque ressource au sein du répertoire communicatif de chaque enfant sourd.

\subsection{Outils de description des relations intersémiotiques}

Pour répondre à cet objectif, dans la limite du découpage réalisé en unités sémantico-syntaxiques sous la ligne enfant, nous proposons l'annotation de trois lignes supplémentaires permettant de décrire les rapports intersémiotiques, la combinatoire intersémiotique et la relation synchronique entre les divers segments composant l'unité.

\subsubsection{Les rapports intersémiotiques}

En nous inspirant des propositions faites par Volterra, Caselli, Capirci \& Pirchio (2005) mais en les adaptant à l'objet de notre étude, nous proposons de caractériser les rapports intersémiotiques de quatre façons.

- Redondant: la redondance s'applique aux combinaisons où les deux modalités sont porteuses d'une information identique. Pour les combinaisons français/LSF, on notera que la redondance s'accompagne d'une structure unique conforme à l'une des deux langues (par exemple dans le cas du français signé).

- Equivalent: cette relation d'équivalence ne s'applique qu'aux combinaisons français/LSF, dans lesquelles les deux messages sont sémantiquement identiques, mais restent syntaxiquement conformes respectivement à chacune des deux langues. Une relation qui, si elle est attestée chez les adultes, ne l'est pas chez les enfants observés jusqu'ici.

- Complémentaire: la complémentarité s'applique aux combinaisons où les deux modalités sont porteuses d'une information différente. Chaque modalité apporte une information spécifique qui n'est pas présente dans l'autre modalité. Le sens n'est alors accessible qu'au niveau de l'unité sémanticosyntaxique prise dans sa globalité.

- Renforçant : ce concept s'applique aux combinaisons où un seul des deux messages est porteur de l'ensemble de l'information. L'autre message n'étant porteur que d'une information partielle au regard de l'information transmise à l'échelle de l'unité.

- Contradictoire: la contradiction s'applique aux combinaisons où ponctuellement une unité entre en contradiction avec l'information véhiculée par la modalité organisatrice. Par exemple lorsque le français organise l'énoncé et que les signes de la LSF produits ponctuellement ne sont pas conformes au contenu sémantique de ce qui est prononcé. Il s'agit donc d'erreurs ou de lapsus, liés à la gestion simultanée de deux systèmes linguistiques.

Sur la base de cette annotation est réalisée ensuite une annotation de toutes les ressources qui interviennent dans l'élaboration du segment sémantique ou de l'unité sous la ligne combinatoire intersémiotique.

\subsubsection{Combinatoire intersémiotique}

Tous les croisements possibles ont été envisagés pour enrichir cette annotation. Dans les productions monolingues ou monomodales, on retrouvera l'une des quatre possibilités majeures: français, onomatopées, LSF ou gestes. Ensuite, les combinaisons seront annotées selon le nombre de croisements :

- combinaison à deux membres : français-onomatopées, français-gestes, français-LSF, etc. ;

- combinaison à trois membres : français-onomatopées-LSF, français-gestes-LSF, etc. ; 
- combinaison à quatre membres : français-onomatopées-LSF-gestes.

\subsubsection{Relations synchroniques}

Enfin, sous la ligne relation synchronique, nous caractérisons la relation synchronique qui régit l'organisation des ressources mobilisées. Ainsi, l'annotation précise si la production est amorcée significativement par une ressource spécifique - i.e " français anticipe », "LSF anticipe », " onomatopée anticipe », « geste anticipe »- ou si les éléments composant le segment sont synchrones.

Par rapport à ce que nous avons déjà précisé et pour exposer l'ensemble de notre modèle d'analyse, il nous reste à expliciter maintenant les annotations détaillées reliées aux trois lignes majeures français, $L S F$ et gestes - la ligne onomatopées n'ayant pas nécessité d'annotations supplémentaires.

\section{Modèle complet hiérarchisé d'analyse}

\subsection{Les annotation du français}

La transcription du français est réalisée sous la ligne français - en proposition ou mots par mots en cas de productions isolées - dans les limites de l'unité sémantico-syntaxique. Les annotations réalisées sur cette base visent à décrire la structure syntaxique des propositions en français. Sous la ligne françaisproposition sont annotés les types de propositions. Nous nous sommes conformées aux catégorisations proposées pour la grille de transcription des enfants entendants (Colletta et al., 2008) qui comprend les types suivants: "phrase nominale », " indépendante », « indépendante présentative », " principale », « principale présentative », « complément de verbe », " complément de phrase », « complément du nom », « complément du nom focalisé », " complément d'adjectif », " complément d'adverbe », " infinitive » et « factitive ». La réalité des productions des enfants sourds nous a amenées à ajouter à cette liste trois items correspondant à des ruptures de langues et/ou de modalités : "syntagme isolé ", "mot isolé » et « thématisation » qui, comme certains de nos exemples l'ont illustré, sont des segments fréquents dans les productions enfantines.

Sur la base du découpage des propos de l'enfant en mots, réalisé automatiquement par la fonction « tokéniser l'acteur » du logiciel ELAN®, nous proposons 3 lignes de transcriptions :

- une ligne d'annotation de la complexité syntaxique : synt.cat.gram., sous laquelle sont annotés les indices de subordination, grâce à un menu déroulant qui contient les items suivants : "conjonction de subordination », « pronom relatif », « préposition », « autres subordonnants »;

- une ligne d'annotation de la complexité discursive disc.cat.gram, sous laquelle sont annotées la présence des connecteurs et des indices anaphoriques, listés de la façon suivante : il s'agit de repérer les éléments assurant un rôle de «connecteur» ou ceux assurant des relations anaphoriques ou cataphoriques (《nom », « déterminant », « pronom », « pronom relatif », « autre ») ;

- une ligne d'annotation de la catégorie syntaxique des mots utilisés : français-type de mots, dont le répertoire est le suivant: "déterminant», «nom», « verbe», «adjectif», «adverbe», «pronom», « préposition », « connecteur », « amalgame », « conjonction », « interjection ».

Toutes ces lignes permettent un traitement quantifié qui sera, de notre avis, d'une très grande utilité pour l'étude et la connaissance des productions en français des enfants sourds.

\subsection{Annotation de la LSF}

Si le découpage des propos en français est assez aisé, le découpage de la LSF en propositions relève d'enjeux théoriques plus complexes liés, d'une part, au fait que les recherches linguistiques sur cette langue sont encore peu développées - et donc parcellaires - et, d'autre part, au fait que ces recherches s'exercent dans des cadres théoriques différenciées souvent peu compatibles les uns avec les autres modèle iconique global pour Cuxac (Cuxac, 2000), modèle fondé sur les grammaires cognitives pour Risler (Risler, 2000) par exemple. Nous nous appuierons donc sur nos propres recherches pour proposer d'une part un découpage de type propositionnel des énoncés ou des messages produits en LSF et d'autre 
part une caractérisation des unités les composant (Bras, Millet \& Risler, 2004 ; Millet, 1997; 2002 ; $2006 \mathrm{a} ; 2006 \mathrm{~b} ; 2008)$. Un autre problème, et non des moindres, tient à la transcription de la langue, chaque chercheur, là encore, mettant au point des transcriptions répondant à ses questionnements et ses objectifs (Dubuisson, 2000 ; Neve, 1992 ; Sallandre, 2003) . $^{14}$.

\subsubsection{Questions liées à la transcription de la LSF}

La difficulté principale tient au fait que le système de transcription doit être à même de rendre compte linéairement des procédés syntaxiques de cette langue qui s'élaborent dans la simultanéité (Vermeerbergen, Leeson \& Crasborn, 2007) et dans l'espace - tridimensionnel par définition - en rendant compte des procédés spécifiques de cette langue.

Les conventions de transcription que nous avons mises au point s'inscrivent dans cette perspective. La LSF étant une langue fondamentalement iconique, elle n'en est pas moins appréhendable sous forme de structures syntaxiques composées d'unités grammaticales. A notre sens, la LSF est, en effet, animée par une dynamique à la fois corporelle et iconique dont il convient de rendre compte dans la transcription syntaxique (Millet, 2002).

On peut par exemple reconnaître dans les constructions narratives deux types de constructions se distinguant par le point de vue adopté par le locuteur, principalement symbolisé par l'implication du corps et du regard. Le locuteur peut maintenir un point de vue externe : il aura alors possiblement recours à des constructions incluant proformes ${ }^{15}$ manuelles mais gardera son statut de narrateur extérieur à la situation décrite - O-VPT Observer's viewpoint chez Mc Neill (1992). Il peut aussi choisir d'adopter un point de vue interne en incarnant le personnage qu'il décrit - C-VPT Character's viewpoint chez Mc Neill. Ce second point de vue correspond à ce que beaucoup de chercheurs en LSF appellent "prise de rôle » (Moody, 1998 [1983]), que Cuxac nomme «transfert personnel» (Cuxac, 2000) et que nous appelons « proforme corporelle» (Bras, Millet, Risler, 2004 ; Millet, 2006). Ces proformes manuelles et corporelles assurent la cohérence syntaxique et discursive, en assurant la continuité référentielle.

Ainsi, la notation [FLEUR pr-fleur, pr-œuf, TOMBER-dedans], qui s'inscrit dans le cas d'une narration faite d'un point de vue externe, correspond à la séquence suivante : le locuteur produit le signe [FLEUR] puis le reprend par une proforme manuelle que, dans un mouvement vers le bas, vient rejoindre la seconde main qui est utilisée en proforme référant à « œuf» : la phrase produite après le signe [FLEUR] s'exécute en un mouvement unique mais contient 4 unités. La phrase [MAMAN OISEAU pr-corp-maman-oiseau, TRICOTER, duratif], inscrite dans un point de vue interne, met en œuvre une proforme corporelle (notée pr-corp) et contient une valeur durative exprimée par la réitération du mouvement exprimant « tricoter ».

Ainsi, au-delà des items lexicaux, toutes les informations signifiantes en LSF sont encodées dans la transcription sous la ligne $L S F$, telles les proformes manuelles et corporelles, les informations verbales, adverbiales, aspectuelles présentes dans le mouvement réalisé. Dans la nécessaire perspective de description multilinéaire de la LSF, nous annotons également la valeur des mimiques dans la transcription

\footnotetext{
${ }^{14}$ Il existe plusieurs systèmes de description/transcription, dont les symboles sont plus ou moins idéographiques par exemple Sign Writing élaboré par Valérie Sutton (http://www.signwriting.org/) ou Hamnosys, élaboré par Siegmund Prillwitz et al. (http://www.sign-lang.uni-hamburg.de/projects/hamnosys.html) mais qui, encodant les dimensions phonologiques des signes ne correspondent pas à nos objectifs descriptifs. De même, les systèmes encodant tous les éléments corporels mobilisés dans la production d'une langue signée - tels Sign stream élaboré à Boston University (http://www.bu.edu/asllrp/SignStream) ou Sign Typ élaboré à l'Université du Connecticut (www.ldc.upenn.edu/signtyp/downloads/) - sont assez éloignés de nos propos qui visent à décrire des pratiques langagières - et non à décrire un/des système(s) linguistique(s).

${ }^{15}$ Les proformes manuelles sont des configurations de main utilisées pour référer à des éléments lexicaux présents dans le discours. Au plan discursif, elles ont une valeur anaphorique ou cataphorique et assument le rôle syntaxique d'un pronom. Elles s'opposent en cela aux spécificateurs de taille et de forme (STF) qui ont une valeur de type adjectivale (caractériser la forme d'un élément). Les dynamiques corporelles et iconiques font qu'un STF peut devenir proforme dès lors qu'il revêt, dans une structure verbale, une valeur pronominale.
} 
de la LSF, sous la ligne mimique $L S F$, selon que la mimique prend une valeur adverbiale, participiale, ou qu'elle confère à la proposition une valeur de négation, ou de modalité de phrase.

\subsubsection{Catégorisation des propositions en LSF}

La catégorisation des propositions effectuée sous la ligne $L S F$-propositions, nous a amenées à penser les indices de complexité syntaxique de manière spécifique. Les types utilisés pour la catégorisation du français n'étant pas applicables tels quels, et les procédés syntaxiques de la LSF n'ayant aucun équivalent en français, nous proposons, pour la LSF, afin de rendre possible une comparaison inter-langues, de distinguer des phrases indépendantes et des phrases complexes. Nous définissons les phrases complexes en LSF au moyen des différents processus qui assurent le lien entre deux propositions. Une proposition peut être liée à une principale par :

- maintien de proforme manuelle

- maintien de proforme corporelle

- spatialisation : un élément spatialisé - ie assigné à un locus ${ }^{16}$ - dans la principale est repris par une proforme ancrée dans ce même locus.

- opposition de locus : chacune des propositions trouve place dans un espace spécifique, le corps, la mimique ou un élément lexical permettant d'exprimer le rapport entre les propositions.

\subsubsection{Annotation des procédés anaphoriques et des catégories syntaxiques en LSF}

Sur la base du découpage réalisé en signes et en unités grammaticales de la LSF, grâce à la fonction «tokéniser l'acteur» du logiciel, l'annotation des propositions en LSF prend place, tout comme l'annotation des propositions en français, sous deux lignes de transcription visant à caractériser la complexité discursive d'une part et l'utilisation des catégories syntaxiques d'autre part.

L'annotation des indices de complexité discursive, sous la ligne disc cat gram LSF, liste les procédés permettant d'assurer la référence en LSF : "proforme manuelle », "proforme corporelle », « locus », «pointé » ${ }^{17}$, «pointé regard». L'item « référence implicite » permet de rendre compte des cas où un STF ou une proforme n'ont pas de référence clairement établie en LSF dans le discours (par exemple l'utilisation de forme de mains caractérisant la forme d'un œuf, sans que le signe [EUF] ait été produit).

Une annotation des catégories syntaxiques auxquelles les signes utilisés appartiennent est effectuée sous la ligne type de signes. Là encore, l'état actuel des connaissances sur la LSF, nous a contraintes à proposer une catégorie « autres » pour identifier les signes qui n'acquièrent pas les statuts grammaticaux suivants : nominal, verbal, adverbial ${ }^{18}$, stf, proforme, proforme double, proforme corporelle, pointage, locus, connecteur ou marque aspectuelle.

Il est à noter que concernant les pratiques enfantines, parce qu'elles manifestent des étapes d'un développement langagier en cours, il est parfois difficile de distinguer, dans la modalité gestuelle, entre LSF et gestes. En effet, comme nous l'avons montré ailleurs (Millet \& Colletta, 1998) les gestes et les signes partagent des matrices communes et cette proximité formelle rend parfois difficile leur distinction. Le critère que nous avons choisi pour parvenir à distinguer les pratiques gestuelles non-verbales et verbales dans les discours enfantins repose sur la présence d'un élément lexical ou d'une structure verbale conforme à la LSF, et ce notamment dans l'élaboration des schémas spatiaux reprenant des configurations

${ }_{17}^{16}$ Le locus est défini comme une portion d'espace supportant une indexation syntaxique permettant la référence.

${ }^{17}$ Le signeur fait référence à un lieu dans l'espace préalablement assigné à une entité du discours par pointage (anaphore) ou, à l'inverse, pointe un espace, le transformant ainsi en un locus où viendra s'ancrer un signe (cataphore). Ces opérations de pointage s'exécutent très majoritairement avec l'index ou avec le regard.

${ }^{18} \mathrm{Il}$ s'agit ici bien plus de statut grammatical que de classes syntaxiques à strictement parler. Le lexique de la LSF étant notionnel, les statuts grammaticaux s'acquièrent en discours mais ne sont pas différenciés en langue, ce qui nous incite ici à la plus grande prudence. Ainsi les notions « fer à repasser » et « repasser » s'incarnent, en lexique, par un signe unique, qui, en discours, va acquérir un statut nominal ou verbal en fonction du contexte d'une part, et de l'investissement corporel d'autre part, toujours requis en cas de statut verbal du signe.

Journal of Language Contact - VARIA 2 (2009)

www. jlc-journal.org 
manuelles, dans un schème phrastique pr-pr-verbe, très fréquent en LSF (Millet, 2002). Lorsque la gestualité n'est pas conforme à ce qui serait attendu en LSF, la description se conformera aux annotations concernant les gestes.

\subsection{Annotation des gestes}

L'étude de la gestualité non-verbale dans les pratiques des locuteurs sourds n'a, à notre connaissance, jamais été étudiée pour elle-même en France ${ }^{19}$. Les études menées sur la gestualité entendante, en revanche abondantes, et notamment dans les pratiques enfantines, ont permis de reconnaitre la gestualité comme une étape de transition vers l'acquisition d'un système langagier verbal. A notre sens, l'observation de la gestualité non-verbale chez les enfants sourds, pourrait permettre de situer l'enfant dans une phase d'acquisition particulière du système linguistique gestuel : la LSF et/ou d'un système linguistique oral. Dans notre corpus, certains enfants sourds accueillis en structure oraliste - i.e. qui n'ont que peu ou pas été exposés à la LSF -, présentent un recours important à la gestualité non-verbale, témoignant de la nécessité de cette ressource langagière pour certains enfants sourds dans l'élaboration de leur vouloir-dire, comme nous l'avons précédemment montré.

Il nous a donc paru important de pouvoir préciser les fonctions des gestes sous une ligne spécifique d'annotation (valeur du geste) pour lesquelles nous proposons la typologie suivante, qui s'appuie principalement, en les remaniant cependant, sur les propositions de catégorisations réalisées pour les adultes et les enfants entendants par Cosnier (1987) et Colletta (2004). Nous proposons, en effet, d'entrer dans le détail des gestes représentationnels au-delà de la seule distinction entre ceux dont la référence est établie dans le contexte immédiat - i.e. déictique - et ceux dont la référence est établie dans la mise en espace de l'univers référentiel - i.e. représentationnel.

\subsubsection{Catégorisation des référentiels exopohoriques}

Les pointages à valeur expohorique, sont classés dans la catégorie « déictique ». Sont entendus comme déictiques, les gestes manuels ou céphaliques de pointage qui font référence à un référent présent dans le contexte immédiat ou à sa direction.

\subsubsection{Catégorisation des représentationnels}

Les gestes référentiels dont la référence est établie dans l'espace présentent des valeurs différentes. Pour parvenir à une description fine des procédés gestuels non-verbaux utilisés par les enfants sourds, ils sont distingués dans les catégories suivantes.

Les «pointages endophoriques » comprennent les gestes manuels ou céphaliques de pointage qui ont une valeur anaphorique ou cataphorique, soit les gestes qui font référence à un locus préalablement assigné à un référent (anaphore), ou postérieurement assigné à un référent (cataphore).

Les «illustratifs» sont les gestes qui représentent les caractéristiques du référent (taille, forme, etc) par configuration manuelle, ou dessinant les caractéristiques du référent dans l'espace.

Les gestes que nous appellerons «spatiographiques» correspondent aux gestes qui assurent une mise en espace de l'univers référentiel. Sont classés dans cette catégorie les gestes qui ont pour fonction de donner une représentation topologique de l'univers référentiel.

Nous distinguons par ailleurs les gestes qui ont pour fonction de représenter l'action d'un référent selon l'implication du corps du locuteur :

- les «mimétiques actions ", qui comprennent les gestes mimant l'action ou le comportement d'un référent par une mise en jeu globale du corps ;

- les «mimétiques trajectoires » qui désignent uniquement les gestes manuels caractérisant le trajet du référent.

\footnotetext{
${ }^{19}$ Quelques chercheurs nord-américains y font référence dans l'analyse de l'interrelation geste/signe - par exemple :
} Emmorey (1999) ou Wilcox (2000). 


\subsubsection{Catégorisations des autres types de gestes}

La typologie des gestes ne saurait être complète sans intégrer les gestes qui n'ont pas valeur référentielle. Nous distinguons à cet effet, les gestes qui peuvent être autonomes :

- les «interactifs» : gestes ayant une fonction phatique à valeur d'appel ou de sollicitation, ou une fonction de régulateur à valeur d'écoute, ou d'attention ;

- les « énonciatifs » : gestes ou mimiques indiquant l’hésitation du locuteur ou la recherche de mots ;

des gestes qui sont généralement co-verbaux :

- les «expressifs»: les gestes qui connotent un contenu de discours qu'ils qualifient : qui situent le contenu par rapport au jugement du locuteur, ou qui assurent ou renforcent la valeur illocutoire de l'énoncé (question, demande de confirmation, etc...).

- les « syntaxiques » : gestes rythmiques accompagnant le flux parolier (beat), marqueurs d'accent mettant en relief une unité linguistique, ou gestes démarcatifs délimitant une unité du discours.

\section{Conclure?}

Il n'est bien évidemment pas question de conclure puisque la grille que nous proposons ici, nous en sommes conscientes, peut subir selon les chercheurs et leurs objectifs bon nombre de modifications et qu'elle n'est, en tout état de cause, qu'un moment de la réflexion. C'est d'ailleurs à ce titre - ouvrir un dialogue scientifique -, qu'il nous a paru intéressant de la proposer en l'état, même si de nombreuses questions restent en suspens, spécialement concernant les annotations liées à la LSF, que les développements de la recherche viendront sans aucun doute modifier.

Il nous semble que, malgré ses inévitables imperfections, elle est à même de servir nos objectifs à court terme : à savoir décrire les conduites narratives des enfants sourds, comprendre la façon dont ils construisent et utilisent un répertoire langagier riche et inventif qui combine langues et modalités au service de leur « vouloir dire».

Nous pensons que cette grille nous permettra également, non pas d'étalonner - compte tenu du faible nombre de sujets qui inscrivent nos recherches dans des démarches qualitatives - mais du moins de poser quelques jalons sur le développement langagier bilingue et bimodal des enfants sourds. Ces résultats pourraient autoriser d'autres lignes didactiques que celles envisagées actuellement qui pensent les sujets sourds - enfants comme adultes au demeurant - en termes dichotomiques, opposant les « sourds oraux » et les « sourds gestuels » (Millet, 2003), alors que nos observations montrent un contact de langue fécond.

Par ailleurs, dans les pistes qui nous paraissent ouvertes par ce travail, on soulignera qu'un encodage systématique d'un nombre conséquent de productions discursives en LSF d'adultes sourds et des outils syntaxiques et discursifs mis en œuvre dans ces discours, permettrait d'avoir une connaissance précise et objective des compétences adultes en LSF, qui manque actuellement cruellement, alors même que l'on s'interroge sur les progressions pédagogiques des enfants ${ }^{20}$.

Ainsi, si cet article posait des bases théoriques et d'ordre plutôt fondamental, les prolongements nous paraissent d'un enjeu humain, social et sociétal d'importance.

\footnotetext{
${ }^{20}$ La LSF ayant été reconnue comme une langue et comme un droit pour les sourds dans la Loi 2005-102 du 11 Février 2005 dite «Loi sur le handicap » et une circulaire du Ministère français de l'Education Nationale (2008109 du 21/08/2008) intitulée "Conditions de mise en æeuvre du programme de la langue des signes française à l'école primaire» fixant ses conditions d'utilisation dans les classes.
} 


\section{Références}

Batista, Aurore. 2008. Enfants sourds intégrés en milieu scolaire "classique" et enfants entendants : une comparaison des productions multimodales d'enfants de 6 et 11 ans. Mémoire de Master. A. Millet (Dir.), Université Stendhal (Grenoble 3).

Bras, Gilles., Agnès Millet \& Annie Risler. 2004. Anaphores et deixis en LSF - Tentative d'inventaire des procédés. Silexicales, $4:$ 57-64.

Colletta, Jean-Marc. 2004. Le développement de la parole chez l'enfant âgé de 6 à 11 ans. Corps, langage et cognition. Bruxelles : Mardaga.

Colletta, Jean-Marc, Aurélie Venouil, Ramona Kunene, Virginie Kaufman \& Jean-Pascal Simon. 2008. Multitrack annotation of child language and gestures. Paper presented at the Language Resources and Evaluation Conference 2008 Workshop on Multimodal Corpora, Marrakech, 26-27 mai 2008.

Conseil de l'Europe. 2005. Cadre européen commun de référence pour les langues. Apprendre. Enseigner. Evaluer. Strasbourg : Didier.

Cosnier, Jacques. 1987. Ethologie du dialogue. In : Jacques Cosnier \& Catherine Kerbrat-Orrechioni (éds.). Décrire la conversation, 291-316. Lyon : Presses Universitaires de Lyon.

Cuxac, Christian. 2000. La langue des signes française - les voies de l'iconicité, Revue Faits de Langues Vol. 15-16. Paris : Ophrys.

Dubuisson, Colette. 2000. Grammaire de la LSQ, 2 tomes. Québec : Université du Québec à Montréal.

Emmorey, Karen.1999. Do signers gesture?. In: Lynn Messing \& Ruth Campbell (eds.). Gesture, Speech and Sign. Oxford: Oxford University Press. (disponible en ligne).

Emmorey, Karen, Helsa Borinstein \& Robin Thompson. 2005. Bimodal bilingualism: code-blending between Spoken English and American Sign Language. In: James Cohen, Kara T. McAlister, Kellie Rolstad \& Jeff MacSwan (eds.). ISB4, Proceedings of the 4th international symposium on bilingualism, 663-673. Somerville MA: Cascadilla Press.

Estève, Isabelle. 2006. Les pratiques communicatives des jeunes adultes sourds - introduction à l'analyse. Mémoire de Master. A. Millet (Dir.), Université Stendhal (Grenoble 3).

Estève, Isabelle. 2007. Analyse des conduites langagières de jeunes adultes sourds. Mémoire de Master. A. Millet (Dir.), Université Stendhal (Grenoble 3).

Estève, Isabelle. 2008. Des pratiques langagières à l'enseignement. Communication présentée au Colloque "Enfance et Plurilinguisme", organisé par Dipralang/Laboratoire de linguistique diachronique, de sociolinguistique et de didactique des langues, Montpellier 3, 26-27 Juin 2008.

Guigas, Lucile. 2006. Représentations sociales et pratiques bilingues de jeunes adultes sourds. Mémoire de Master. A. Millet (Dir.), Université Stendhal (Grenoble 3).

Loncke, Filip, Sophie Quertinmont, Kathleen Martens \& Inge Dussart. 1996. Les jeunes sourds et la pratique de la communication bimodale. In : Christiane Lepot-Froment \& Nadine Clerebaut (éds.), L'enfant sourd, 317-346. Bruxelles : De Boeck.

Lucas, Ceil. 1996. Phénomènes de contact dans les communautés sourdes. In : Colette Dubuisson \& Denis Bouchard (éds.). Spécificités de la recherche linguistique sur les langues signées. Montréal : Les cahiers scientifiques de l'Association canadienne française pour l'avancement des sciences, 89 : 107-116.

Lucas, Ceil. 2001. The sociolinguistics of Sign Languages. Cambridge: Cambridge University Press. 
Lucas, Ceil, Robert Bayley \& Clayton Valli. 2001. Sociolinguistic variation in American sign language. Washington, D.C.: Gallaudet University Press.

Lüdi, Georges \& Bernard Py. 1986. Etre bilingue. Bern : Peter Lang.

Mc Neill, David. 1992. Hand and Mind, what gestures reveal about thought. Chicago: University of Chicago Press.

Millet, Agnès. 1997. Réflexions sur le statut du mouvement en LSF - aspects lexicaux et syntaxiques. Lidil (Revue de linguistique et de didactique des langues), 15 : 11-30.

Millet, Agnès. 2002. Les dynamiques iconiques et corporelles en LSF. Lidil, 26 : 27-44.

Millet, Agnès. 2003. Les représentations de la LSF - Comment penser un sujet sourd bilingue et biculturel. Nouvelle Revue de l'AIS - Adaptation et Intégration Scolaire, 23 : 63-72. Edition du Centre national d'études et de formation pour l'enfance inadaptée.

Millet, Agnès. 2006a. Dynamiques iconiques en LSF aspects syntaxiques et discursifs, In Daniel Daigle \& Anne-Marie Parisot, (éds.). Surdité et société, 129-142. Montréal: Presses universitaires du Québec.

Millet, Agnès. 2006b. Le jeu syntaxique des proformes et des espaces dans la cohésion narrative en LSF. Glottopol 7 - Revue en ligne : http://www.univ-rouen.fr/dyalang/glottopol/telecharger/numero $7 / \mathrm{gpl} 17$ 00presentation.pdf .

Millet, Agnès. 2007. Bilingual cross-modal communicative practices of young deaf adults. Paper presented at the 6th International symposium on bilingualism. ISB6. Hamburg, May 30-June 2, 2007.

Millet, Agnès. 2008. L'expression de la quantité définie et indéfinie en LSF. Silexicale, 5 : 77-89.

Millet, Agnès \& Jean-Marc Colletta. 1998. Gestualité référentielle des entendants et LSF : des matrices communes ?. Congrès International des Linguistes, 20-25 Juillet 1997. Paris : CD-Rom, Pergamon - Elsevier.

Millet, Agnès. \& Isabelle Estève. 2008. Pratiques langagières bilingues et multimodales de jeunes adultes sourds. Actes en ligne du Colloque Journées d'Etude de la Parole. www.afcpparole.org/doc/Archives JEP/2008 XVII JEP Avignon/PDF/avignon2008 pdf/JEP/029 jep 1 $\underline{564 . p d f}$

Millet, Agnès, Isabelle Estève \& Lucile Guigas. 2008. Pratiques communicatives de jeunes sourds adultes. Rapport pour la Délégation Générale à la Langue Française et aux Langues de France. Exemplaire photocopié. Laboratoire Lidilem.

Moody, Bill. (1998 [1983]). La langue des signes - Histoire et grammaire. Vincennes : IVT Editions.

Neve, François-Xavier. 1992. Phonologie ou gestématique des langues des signes des sourds : gestèmes, allogestes et neutralisations ?. La linguistique, 28-1:69-93.

Risler, Annie. 2000. La langue des signes française, langue iconique. Ancrage perceptivo-pratique des catégories du langage et localisme cognitif à travers l'étude de la motivation des signes et de la spatialisation des relations sémantiques. Thèse de doctorat. J.L. Nespoulous (Dir.). Université de Toulouse Le Mirail.

Sallandre, Marie-Anne. 2003. Les unités du discours en Langue des Signes Française. Tentative de catégorisation dans le cadre d'une grammaire de l'iconicité. Thèse de doctorat. C. Cuxac (Dir.). Université Paris 8. 
Séro-Guillaume, Philippe (éd.). 1994. Les sourds, le français et la langue des signes. Bulletin du Centre CNEFEJS (Centre national de formation des enseignants intervenant auprès de jeunes sourds). Actes du séminaire du 21-25 février 1994. Chambéry : Université de Savoie.

Vermeerbergen, Myriam, Lorraine Leeson \& Onno Crasborn. 2007. Simultaneity in signed language: form and function. Amsterdam - Philadelphie: John Benjamins.

Volterra, Virginia, Maria-Cristina Caselli, Olga Capirci \& Sabine Pirchio. 2005. Le rôle des gestes dans l'acquisition du langage chez les enfants entendants, les enfants non entendants et les enfants au développement atypique. Catherine Transler, Jacqueline Leybaert \& Jean-Emile Gombert (éds.). L'acquisition du langage par l'enfant sourd, 89-120. Marseille : Solal.

Vonen, Arnfinn Muruvik, Arnesen, Knut, Enerstvedt, Regi Theodor \& Anne Varran Nafstad. 1996. Bilingualism and literacy concerning Deafness and Deaf-blindness - Proceeding of an International Workshop. Oslo: Skadalen Publication.

Wilcox, Sherman. 2000. Gesture and Language. Gesture, 4-1: 43-73. 
Annexe : Grille hiérarchisée d'annotation sous le logiciel ELAN ${ }^{\circledR}$

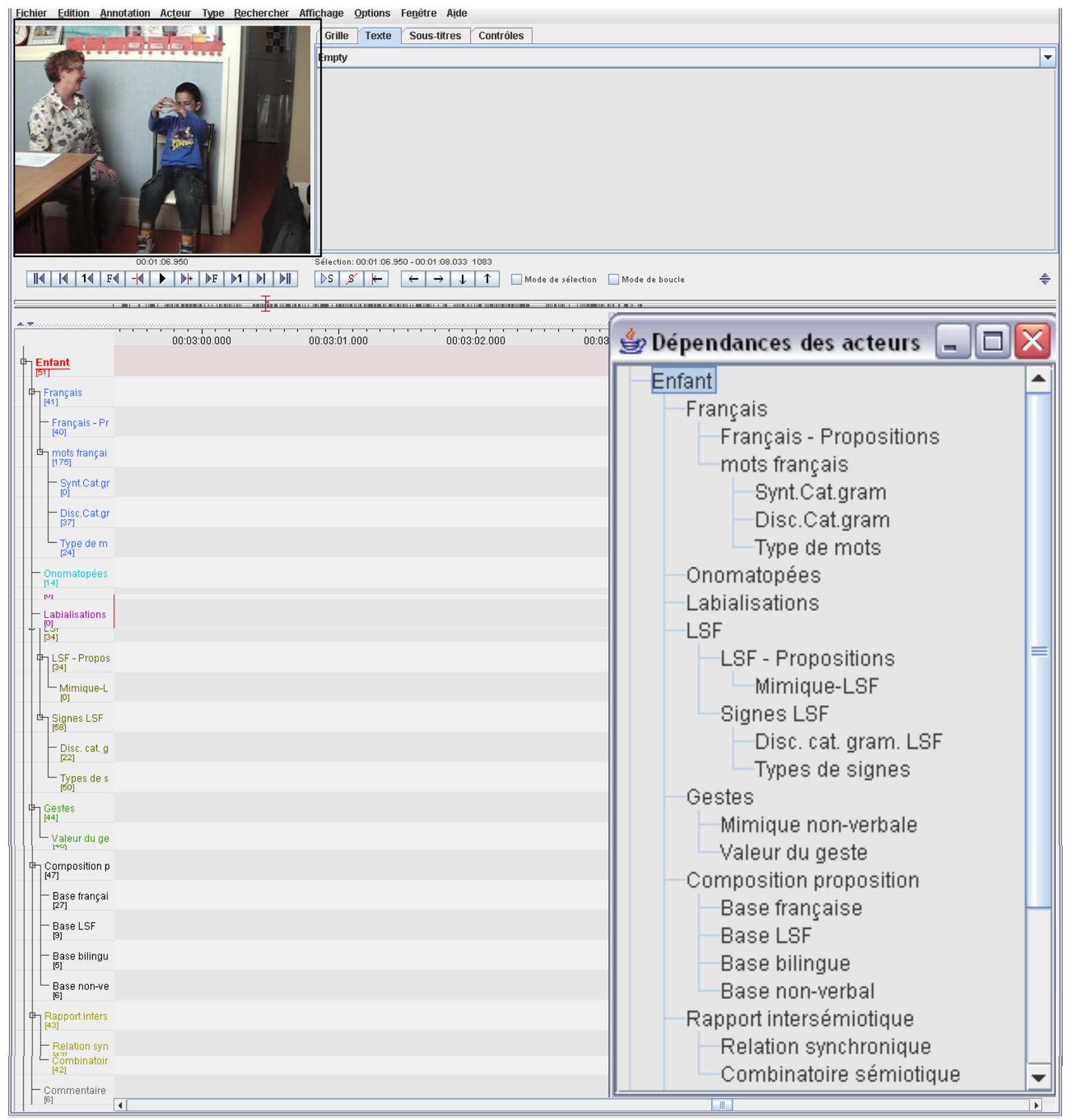


